\title{
Significado clínico de la neoplasia intraepitelial prostática y de la proliferación acinar focal atípica: relación con el cáncer de próstata
}

\author{
Pascual Mateo C*, Luján Galán M*, Rodríguez García N**, Llanes González L**, \\ Berenguer Sánchez A**.
}

*Servicio de Urología. Hospital Infanta Cristina Parla. Madrid.

**Servicio de Urología. Hospital de Getafe. Madrid.

Actas Urol Esp. 2008;32(7):680-685

\begin{abstract}
RESUMEN
SIGNIFICADO CLÍNICO DE LA NEOPLASIA INTRAEPITELIAL PROSTÁTICA Y DE LA PROLIFERACIÓN ACINAR FOCAL ATÍPICA: RELACIÓN CON EL CÁNCER DE PRÓSTATA

Introducción: El papel de la neoplasia intraepitelial prostática (PIN) y de la proliferación acinar focal atípica (ASAP) en el marco de una biopsia transrrectal todavía no se encuentra por completo definido; aunque ambas lesiones han sido consideradas clásicamente premalignas, hoy en día la necesidad de la rebiopsia sistemática sigue siendo controvertido.

Objetivos: En este trabajo hemos estudiado el papel de estas lesiones y su relación con el cáncer de próstata.

Material y métodos: Se incluyeron 138 sujetos (108 PIN, 30 ASAP) a los que se le practicó rebiopsia al 67\%; la tasa de cáncer en la rebiopsia inmediata fue del 19 y $27 \%$ respectivamente sin identificar ningún factor clínico para predecir cáncer en la rebiopsia (PSA, edad, tacto rectal, volumen prostático).

Resultados: Durante el seguimiento, la mayor tasa de cáncer se observó en el ASAP, seguido del PIN y finalmente de las lesiones benignas; el único predictor clínico-patológico independiente de cáncer en este caso fue la existencia de ASAP en la primera biopsia transrrectal.

Conclusiones: La necesidad de rebiopsiar sistemáticamente a los sujetos con PIN en la biopsia de próstata ha sido puesta en duda en la literatura reciente, mientras que la necesidad de rebiopsiar los ASAP sigue estando claramente indicado.
\end{abstract}

Palabras claves: Cáncer de próstata. Neoplasia intraepitelial prostática. Proliferación acinar pequeña atípica. Biopsia transrrectal.

\begin{abstract}
CLINICAL SIGNIFICANCE OF PROSTATIC INTRAEPITHELIAL NEOPLASM AND ATYPICAL SMALL ACINAR PROLIFERATION: RELATIONSHIP WITH PROSTATE CANCER

Introduction: Prostatic intraepithelial neoplasia (PIN) and atypical small acinar proliferation (ASAP) in the setting of prostatic needle biopsies are considered premalignant although questions still remain.

Objetives: In this paper, we have studied the clinical relevance of these histologic findings.

Material and methods: We collected 138 subjects (108 PIN, 30 ASAP); in 67\% we performed a second biopsy and the rate of cancer in this late biopsy were $19 \%$ and $27 \%$ respectively. We cannot identify any clinical factor to predict the finding of cancer in the re-biopsy (PSA, age, digital rectal examination, prostatic volume).

Results: In the follow-up, we observed higher rates of cancer for the ASAP; the finding of ASAP was the single clinical or histopathological factor that was an independent predictor of cancer.

Conclusions: We observed that the finding of ASAP was an indication for re-biopsy because of the higher rates of cancer; on the contrary, the paper of PIN in the prostatic needle biopsy still requires further investigation.
\end{abstract}

Keywords: Prostate cancer. Prostatic intraepithelial neoplasm. Atypical small acinar proliferation. Prostate needle biopsies.

$\mathrm{L}$ a biopsia de próstata (transrectal o perineal) juega un papel básico en el abordaje diagnóstico del cáncer de próstata (CP). Hoy en día, la principal indicación contemporánea es la elevación del antígeno específico prostático (PSA). El mayor número de determinaciones de PSA ha conducido a un mayor número de biopsias y con ello una mayor detección de cánceres así como de lesiones que, lejos de ser benignas, tampoco alcanzan criterios para ser enmarcadas como adenocarcinomas. El significado clínico de estas lesiones (supuestamente relacionadas con el CP) ha cobrado mayor importancia, ya que 
implican la necesidad de repetición de la biopsia o de nuevas estrategias para la misma. Hoy en día, los patólogos tienden a uniformar y simplificar el informe patológico de la biopsia de próstata con aguja, de manera que sea lo más conciso e inequívoco posible; el grupo de patólogos del European Randomized Study of Screening for Prostate Cancer (ERSPC) estableció unos criterios para informar de hasta ocho tipos de lesiones: benignas, inflamación aguda, inflamación granulomatosa crónica, atrofia glandular, neoplasia intraepitelial, adenocarcinoma, lesiones sospechosas de malignidad, y otros tumores prostáticos ${ }^{1}$. Las más controvertidas en cuanto a su posible asociación con el CP son las siguientes:

Neoplasia intraepitelial prostática (PIN) de alto grado: Se define como acinos y conductos prostáticos arquitecturalmente benignos pero cubiertos por células atípicas. Estas células atípicas comparten cambios genéticos, morfológicos, histológicos e inmunohistoquímicos con el $\mathrm{CP}$, pero carecen de la invasión de la membrana basal. Es una lesión histológica existente en un 2,7 a 14,2\% de las biopsias de próstata ${ }^{2-4}$. Tradicionalmente se ha considerado a esta lesión como un factor de riesgo para $\mathrm{CP}$ y por ello la identificación de PIN de alto grado sin CP concomitante ha determinado la indicación de una rebiopsia inmediata. Algunos trabajos postulaban que esta lesión suponía un riesgo relativo de 15 veces de asociar cáncer ${ }^{5}$ y se ha publicado una tasa de coexistencia de PIN con cáncer de próstata de hasta el $85 \%{ }^{6}$. El PIN no eleva significativamente las cifras de PSA sérico total ni libre ya que es secretado hacia los ductos prostáticos (integridad estructural de la membrana basal). La tasa de detección de cáncer de próstata en la re-biopsia de PIN ha variado en el tiempo con una clara tendencia descendente ${ }^{7}$, e incluso con evidencia en la actualidad de que un PIN de alto grado no supone un mayor riesgo de presentar $\mathrm{CP}^{8}$.

Proliferación acinar pequeña atípica (atypical small acinar proliferation, ASAP): Aunque es la nomenclatura más encontrada en la literatura, esta denominación es en sí misma controvertida. Actualmente se recomienda no hablar de proliferación acinar pequeña atípica (ASAP) o proliferación acinar atípica (AAP) y emplear en su lugar la denominación de lesiones sospechosas pero no diagnósticas de adenocarcino- $\mathrm{ma}^{1}$. Se engloban aquí aquellas lesiones demasiado pequeñas o en las que faltan suficientes criterios para ser capaz de hacer un diagnóstico definitivo de adenocarcinoma. La mitad de los casos de ASAP son realmente carcinomas biopsiados en su margen, y la otra mitad representan cambios reactivos benignos, acinos prostáticos atróficos con atipias, o bien hallazgos indeterminados que no pueden descartar por completo el diagnóstico de carcinoma ${ }^{9}$; es decir, el diagnóstico de ASAP indica al urólogo que la biopsia muestra características histológicas ni claramente benignas ni malignas y que requiere o bien un estrecho seguimiento clínico o bien una repetición de la biopsia, habitualmente ampliando la estrategia de la misma (habitualmente con la toma de cilindros adicionales). En la literatura, la tasa de $\mathrm{CP}$ en la re-biopsia de sujetos con ASAP ha oscilado entre un 30 y un $60 \%{ }^{10}$.

El objetivo de nuestro trabajo ha sido establecer el significado clínico de la neoplasia intraepitelial de alto grado (PIN alto grado) y de la proliferación acinar focal atípica (ASAP) en nuestra serie.

\section{MATERIAL Y MÉTODOS}

Se ha llevado a cabo un análisis retrospectivo en el que se incluyeron 138 sujetos con el diagnóstico en la primera biopsia (randomizada sextante ecodirigida) de PIN ( $n=108)$ o ASAP ( $n=30)$, en los que se indicó de manera sistemática una repetición de dicha biopsia (realizada nuevamente de modo sextante). Se registraron algunos factores que podrían tener influencia sobre la detección de $\mathrm{CP}$ tras la repetición de la biopsia: edad, el tacto rectal, el nivel de PSA (ng/ml) y el volumen ecográfico (cc) en el momento de la biopsia.

El trabajo incluyó dos partes: en la primera se identificó la tasa de cáncer en la re-biopsia inmediata de las lesiones estudiadas (evaluando así la existencia de CP concomitante) así como las variables clínicas capaces de predecir el resultado de la dicha re-biopsia. En una segunda parte se analizó la tasa de cáncer registrada en el seguimiento a medio y largo plazo de estos pacientes (esto es, la aparición de CP a lo largo de la evolución posterior), añadiendo un tercer grupo de 363 varones con biopsia con resultado de benignidad como grupo control. También se estudiaron las potenciales variables predictoras clínico-patológicas del desarrollo de cáncer en la evolución de estos sujetos. 
El análisis estadístico empleó pruebas de comparación de medias (chi-cuadrado, t de Student, U de Mann-Whitney), análisis de supervivencia Kaplan-Meier y análisis de regresión de Cox. Se empleó el paquete estadístico SPSS 13 para el procesamiento de los datos.

\section{RESULTADOS}

De los 138 sujetos con PIN o ASAP en los que se indicó repetición de la biopsia, aceptaron la misma 92 sujetos $(66,67 \%)$. Once $(8 \%)$ fueron sometidos a una tercera biopsia y sólo un sujeto $(0,75 \%)$ accedió a realizar la cuarta biopsia.

Primera parte (detección de CP en la rebiopsia inmediata)

La edad media de estos pacientes fue de 63,8 años (IC95\% 62,7-65,0, mediana 63 años) y su nivel medio de PSA fue de 6,17 ng/ml (IC95\% 5,33-7,00, mediana 4,93). El tacto rectal fue sospechoso en un 9,4\% y el volumen ecográfico medio fue de 46,77 cc (IC95\% 42,1-51,5). No se identificaron diferencias significativas en cuanto a edad, tacto rectal, PSA ni volumen ecográfico entre los grupos de pacientes con PIN y ASAP. De los 92 sujetos en los que se consiguió re-biopsia, en $19(20,7 \%)$ se detectó CP. De los 108 pacientes con PIN se repitió la biopsia en 69 (64\%), con una tasa de detección de CP de 18,8\% (Fig. 1). De los 30 pacientes con ASAP se re-biopsiaron 23 (76,7\%), detectando CP en un 26,9\% (Fig.2). No se observaron diferencias estadísticamente significativas en la tasa de detección de cáncer de próstata en la re-biopsia entre los sujetos con PIN o ASAP.

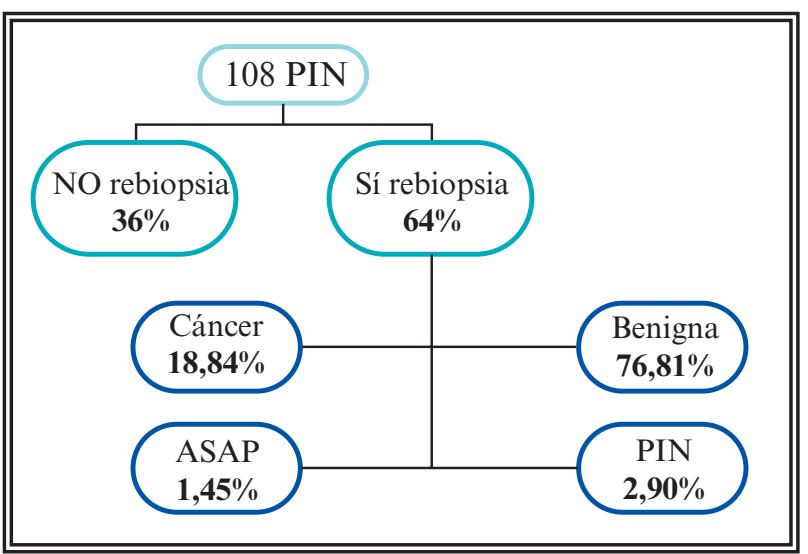

FIGURA 1. Resultado de la rebiopsia de las neoplasias intraepiteliales prostáticas.

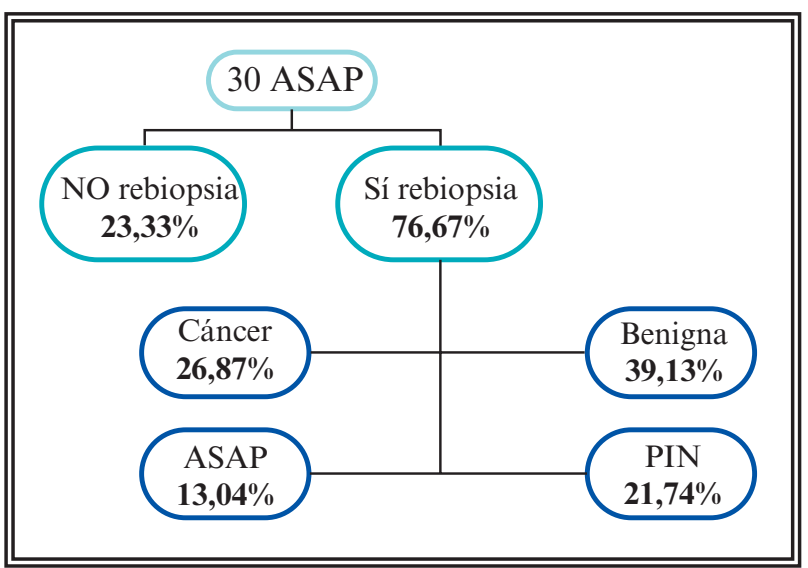

FIGURA 2. Resultado de la rebiopsia de las proliferaciones acinares focales atipicas.

Segunda parte (implicación de estas lesiones supuestamente premalignas en la detección de CP el seguimiento)

Para el análisis Kaplan-Meier (evento definido como detección de CP en el seguimiento) se emplearon 501 sujetos (108 PINes, 30 ASAP, 363 biopsias benignas). El tiempo de seguimiento medio fue de 16,6 meses (IC95\% 14,4-18,9). La curva de supervivencia global (tomando como evento la tasa de detección de cáncer) se muestra en la Figura 3. Las curvas de supervivencia para detección de cáncer según la lesión histológica se muestran en la Figura 4. Los

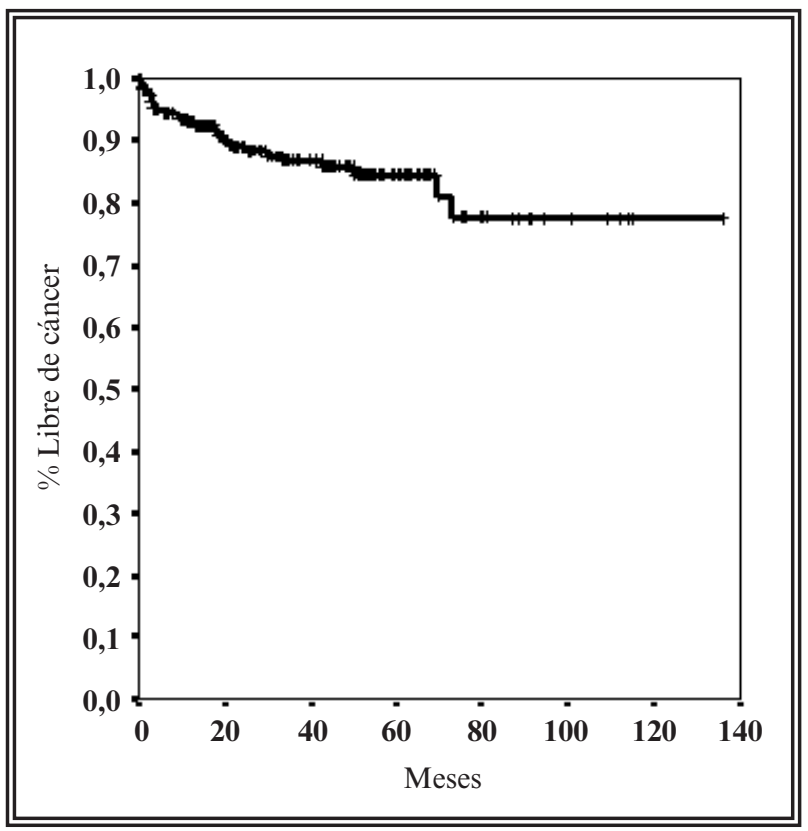

FIGURA 3. Curva de Kaplan-Meier global de toda la serie de tasa libre de cáncer en pacientes con una lesión premaligna en la biopsia transrrectal. 


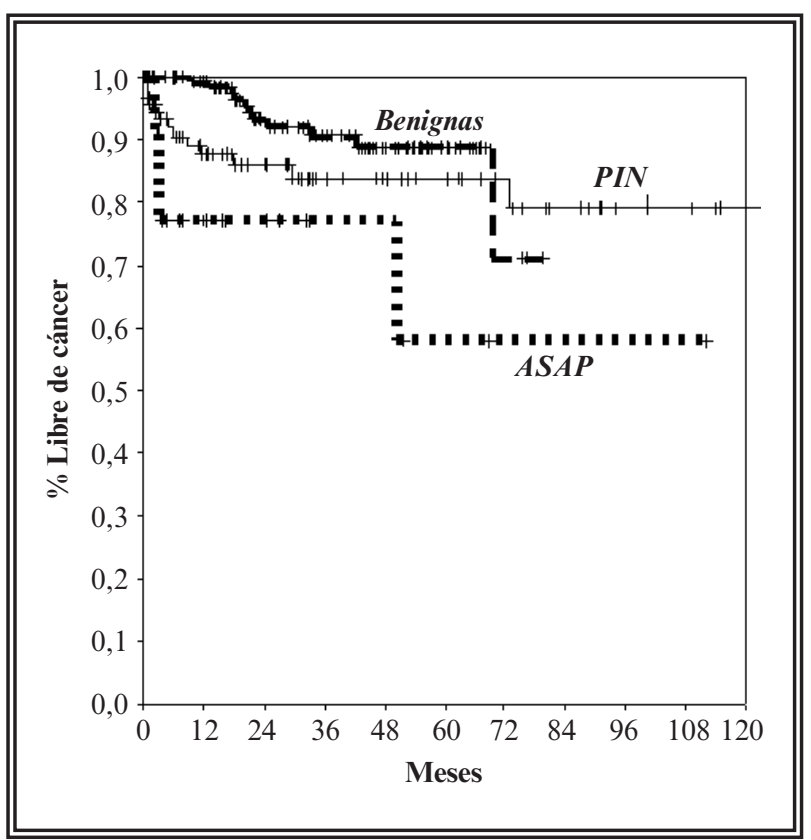

FIGURA 4. Curvas de Kaplan-Meier de los distintos tipos histológicos.

sujetos con ASAP son los que muestran una mayor detección de CP durante el seguimiento, mientras que los PINes tienen tasas de detección intermedias entre ASAP y biopsias benignas; estas diferencias son estadísticamente significativas $(\mathrm{p}=0.0081)$. La tasa libre de cáncer a los 2 y 5 años fue del $88.35 \%$ y $81 \%$ para toda la serie; a los 2 años estaban libres de cáncer el $92 \%$ de los sujetos con biopsia negativa, el $85 \%$ de los sujetos con biopsia de PIN y el $62 \%$ de los sujetos con ASAP.

Finalmente, analizamos las variables predictoras de cáncer tanto en la re-biopsia inmediata como en el seguimiento. Dada la ausencia de diferencias significativas en el análisis univariante de las variables clínico-patológicas de la re-biopsia inmediata, carece de sentido diseñar un análisis multivariante mediante una regresión de Poisson. Se llevó a cabo un análisis de regresión de riesgos proporcionales de Cox para identificar las variables clínico-patológicas predictoras de cáncer en el seguimiento (edad, psa, diagnostico primario de la biopsia transrrectal); de todas las variables introducidas, la única que mostró tener capacidad predictiva significativa estadísticamente fue la presencia de ASAP en la primera biopsia transrectal que multiplica las posibilidades de diagnosticar posteriormente un cáncer durante el seguimiento por 4,41 (IC95\% 1,623-12, $\mathrm{p}=0,004)$.

\section{DISCUSIÓN}

En este trabajo se ha estudiado el papel que tiene en nuestro entorno la presencia en una biopsia de próstata la existencia de PIN de alto grado y de ASAP. Hemos evidenciado que el PIN de alto grado no introduce un mayor riesgo de presentar $\mathrm{CP}$ concomitante ni en la evolución futura, al contrario que el hallazgo de ASAP.

La relación del PIN de alto grado con el CP es un hecho bien establecido y que ha sido objeto de revisiones como las de Bostwick et al y las de Haggman et $\mathrm{al}^{11,12}$. Actualmente es un hecho universalmente aceptado que el PIN de bajo grado no debe ser comunicado en los informes histopatológicos por la gran variabilidad interobservador y por la dificultad para diferenciarlo del tejido prostático benigno. Además, el riesgo de $\mathrm{CP}$ concomitante en esta lesión es equiparable a la del tejido benigno ${ }^{13}$. La incidencia de PIN de alto grado en biopsias prostáticas oscila entre el 4 y el $8 \%^{10}$. El diagnóstico de PIN de alto grado tiene un cierto grado de subjetividad y por ello la reproducibilidad diagnóstica interobservador mejora en los uro-patólogos y disminuye en los patólogos generales ${ }^{14,15}$.

La importancia clínica del PIN de alto grado radica en su estrecha relación con el adenocarcinoma prostático; sin embargo, el sentido de esta relación ha sufrido modificaciones con el tiempo. Los primeros estudios llevados a cabo en los años 80 y 90 mostraban tasas elevadas de cáncer en la re-biopsia, en torno a un 50\% ${ }^{5,16-20}$. Por el contrario, los trabajos más recientes comunican tasas muy inferiores, entre un $20-25 \%^{21-25}$, o incluso inferiores ${ }^{8}$.

En nuestra serie actual el riesgo de cáncer de próstata en la re-biopsia inmediata de los PIN de alto grado fue de 18,8\% (parecida al riesgo de detectar CP en una población sin biopsia previa con PSA elevado), muy similar a los resultados publicados en la literatura más reciente y significativamente menor a los datos publicados en nuestro centro en el año 2000 en el que se comunicó una tasa del $40,7 \%{ }^{7}$. En general, los trabajos con mayor número de pacientes muestran menores tasas de cáncer de próstata. En la revisión de Epstein et $\mathrm{al}^{10}$, la mediana de tasa de cáncer en la re-biopsia de los 33 trabajos incluidos fue $24,1 \%$, observando que la mayoría de los publicados a partir del 2000 tenían tasas de cáncer menores a la mediana. Por otro lado, el riesgo de cáncer en la re-biopsia de sujetos con 
biopsia previa benigna fue de $18,7 \%^{10}$. En general, los trabajos más recientes no han demostrado diferencias significativas en la re-biopsia de sujetos con biopsias de próstata benignas o de un PIN de alto $\operatorname{grado}^{5,23,26,27}$. En nuestro estudio, la mayor parte de los tumores se diagnosticaron en la primera re-biopsia, siendo la rentabilidad de las re-biopsias adicionales muy baja para el diagnóstico de cáncer. En relación con esto, la mayor parte de los trabajos confirman estos hallazgos. Igualmente, la mayor parte de los trabajos confirman nuestros hallazgos de que ninguna de las variables clínicas estudiadas (PSA, tacto rectal, edad) permite predecir el resultado de la re-biopsia de un PIN de alto grado.

Una de los puntos conflictivos actuales se centra en la tasa de cáncer detectado en la re-biopsia según el número de cilindros obtenidos. En nuestro centro tanto para la biopsia inicial como para las rebiopsias se obtuvieron 6 cilindros; en la literatura, los trabajos con el mismo esquema comunicaban una tasa media de cáncer del 14\%, mientras que en aquellos que aumentaban los cilindros en la rebiopsia, la tasa de cáncer aumentaba hasta casi el $32 \%$. Cuando el número de cilindros era de 8 o superior desde el primer momento, la tasa en la rebiopsia descendía al 14,6\% debido a la mayor detección de cánceres en la primera biopsia. Esto hace que, disponiendo de protocolos de muestreo más exhaustivos, los sujetos con PIN de alto grado no están sometidos a mayor riesgo de cáncer que los sujetos con biopsia benigna ${ }^{10}$.

Una de las facetas estudiadas en este trabajo fue la capacidad de que un sujeto con PIN de alto grado desarrolle cáncer en el seguimiento. En este sentido no encontramos diferencias entre los sujetos con PIN y con biopsias benignas; no obstante, la historia natural del PIN de alto grado todavía es desconocida y existen algunos trabajos que afirman que a largo plazo aumenta la tasa de detección de $\mathrm{CP}^{4}$. Por tanto, el papel del PIN en la biopsia de próstata se encuentra en evolución y parece necesario estudios centrados en esta entidad para aclarar su historia natural.

Como ya se ha comentado, el la proliferación acinar focal atípica o ASAP refleja un vasto grupo de lesiones de significado clínico variable. Se considera que la incidencia de diagnostico de ASAP oscila en torno al 5\%. En nuestro trabajo hemos incluido un total de 30 pacientes con ASAP en la biopsia inicial, encontrando $\mathrm{CP}$ en la re-biopsia inmediata en $27 \%$. Este porcentaje es menor del publicado en la literatura que oscila en torno al 40\% ${ }^{23,28-30}$. La mayor parte de los cánceres diagnosticados, lo fueron en la primera re-biopsia, de modo similar a otros trabajos revisados ${ }^{22,29,31,32}$. Del mismo modo que con los PIN de alto grado, el protocolo empleado en cuanto al número y localización de los cilindros influye en la tasa de cáncer detectado en la re-biopsia; en la literatura se ha publicado que una biopsia selectiva sobre la zona de ASAP aumenta el rendimiento para la detección de cáncer en la re-biopsia hasta un 48-85\%. Muchos autores recomiendan aumentar el número de cilindros en la re-biopsia de un $\operatorname{ASAP}^{32,33}$.

En conclusión, se puede afirmar que el hallazgo en una biopsia de próstata de un atipia focal sospechosa de cáncer debe ir seguido de manera sistemática de una re-biopsia así como de un estrecho seguimiento dada la alta tasa de cáncer tanto a corto como a largo plazo. El papel de la neoplasia intraepitelial de alto grado se mantiene controvertido, pero a la luz de los datos analizados se puede concluir que su identificación en una biopsia no implicaría la necesidad de una re-biopsia sistemática inmediata. No obstante, siguen siendo necesarios estudios más detallados que nos permitan conocer la historia natural completa de esta entidad.

\section{REFERENCIAS}

1. Van der Kwast TH, Lopes C, Santonja C, Pihl CG, Neetens I, Martikainen $\mathrm{P}$, et al. Guidelines for processing and reporting of prostatic needle biopsies. J Clin Pathol. 2003;56(5):336-340.

2. Abdel-Khalek M, El-Baz M, Ibrahiem el-H. Predictors of prostate cancer on extended biopsy in patients with high-grade prostatic intraepithelial neoplasia: a multivariate analysis model. BJU Int. 2004;94(4):528-533.

3. Alsikafi NF, Brendler CB, Gerber GS, Yang XJ.. High-grade prostatic intraepithelial neoplasia with adjacent atypia is associated with a higher incidence of cancer on subsequent needle biopsy than high-grade prostatic intraepithelial neoplasia alone. Urology. 2001;57(2):296-300.

4. Lefkowitz GK, Taneja SS, Brown J, Melamed J, Lepor H. Followup interval prostate biopsy 3 years after diagnosis of high-grade prostatic intrapeithelial neoplasia is associated with high likelihood of prostate cancer, independent of change in prostate specific antigen levels. J Urol. 2002;168(4 Pt 1):1415-1418.

5. Davidson D, Bostwick DG, Qian J, Wollan PC, Oesterling JE, Rudders RA, et al. Prostatic intrepithelial neoplasia is a risk factor for adenocarcinoma: predictive accuracy in needle biopsies. J Urol. 1995;154(4):1295-1299.

6. Qian J, Wollan P, Bostwick CG. The extent and multicentricity of high-grade prostatic intraepithelial neoplasia in clinically localized prostatic adenocarcinoma. Hum Pathol. 1997;28(2):143148. 
7. Luján Galán M, Páez Borda A, Romero Cajigal I, Gómez de Vicente JM, Berenguer Sánchez A. Significado clínico de la neoplasia intraepitelial prostática. Arch Esp Urol. 2000;53(3):227229.

8. Vis A, Hoedemaeker RF, Roobol M, Schröder FH, van der Kwast TH. The predictive value for prostate cancer of lesions that raise suspicion of concomitant carcinoma. Cancer. 2001;92(3): 524-34.

9. Schlesinger C, Bostwick DG, Iczkowski KA. High-grade prostatic intraepithelial neoplasia and atypical small acinar proliferation. Am J Surg Pathol. 2005;29(9):1201-1207.

10. Epstein JI, Herawi M. Prostate needle biopsies containing prostatic intraepithelial neoplasia or atypical foci suspicious for carcinoma: implications for patient care. J Urol. 2006;175(3 Pt 1):820-834.

11. Bostwick DG, Qian J. High-grade prostatic intraepithelial neoplasia. Mod Pathol. 2004;17(3):360-379.

12. Haggman MJ, Macoska JA, Wojno KJ, Oesterling JE. The relationship between prostatic intraepithelial neoplasia and prostate cancer critical issues. J Urol. 1997;158(1):12-22.

13. Goeman L, Joniau S, Ponette D, Van der Aa F, Roskams T, Oyen R, et al. Is low-grade prostatic intraepithelial neoplasia a risk factor for cancer?. Prostate Cancer Prostatic Dis. 2003;6 (4):305-310

14. Epstein JI, Grignon DJ, Humphrey PA, McNeal JE, Sesterhenn IA, Troncoso P, et al. Interobserver reproducibility in the diagnosis of prostatic intraepithelial neoplasia. Am J Surg Pathol. 1995; 19(8):873-886.

15. Allam CK, Bostwick DG, Hayes JA, Upton MP, Wade GG, Domanowski GF, et al. Interobserver variability in the diagnosis of high-grade prostatic intraepithelial neoplasia and adenocarcinoma. Mod Pathol. 1996;9(7):742-751.

16. Brawer MK, Bigler SA, Sohlberg OE, Nagle RB, Lange PH. Significance of prostatic intraepithelial neoplasia on prostate needle biopsy. Urology. 1991;38(2):103-107.

17. Aboseif S, Shinohara K, Weidner N, Narayan P and Carroll PR. The significance of prostatic intra-epithelial neoplasia. $\mathrm{Br} \mathrm{J}$ Urol. 1995;76(3):355-359.

18. Perachino M, di Ciolo L, Barbetti V, Ardoino S, Vitali A, Introini C, et al. Results of rebiopsy for suspected prostate cancer in symptomatic men with elevated PSA levels. Eur Urol. 1997;32 (2): 155-159

19. Weinstein MH, Epstein JI. Significance of high-grade prostatic intraepithelial neoplasia on needle biopsy. Hum Pathol. 1993; 24(6):624-629

20. Keetch DW, Humphrey P, Stahl D, Smith DS, Catalona WJ. Morphometric analysis and clinical followup of isolated prostatic intraepithelial neoplasia in needle biopsy of the prostate. $\mathrm{J}$ Urol. 1995;154(2 Pt 1):347-351.

21. Lefkowitz GK, Sidhu GS, Torre P, Lepor H, Taneja SS. Is repeat prostate biopsy for high-grade prostatic intraepithelial neoplasia necessary after routine 12-core sampling?. Urology. 2001;58 (6):999-1003.
22. Moore CK, Karikehalli S, Nazeer T, Fisher HAG, Kaufman RP, Mian BM: Prognostic significance of high grade prostatic intraepithelial neoplasia and atypical small acinar proliferation in the contemporary era. J Urol. 2005;173(1):70-72.

23. Postma R, Roobol M, Schroder FH, van der Kwast TH. Lesions predictive for prostate cancer in a screened population: first and second screening round findings. Prostate. 2004;61(3): 260-266.

24. Algaba F. Evolution of isolated high-grade prostate intraepithelial neoplasia in a Mediterranean patient population. Eur Urol. 1999; 35(5-6):496-497.

25. Kronz JD, Allan CH, Shaikh AA, Epstein JI. Predicting cancer following a diagnosis of high-grade prostatic intraepithelial neoplasia on needle biopsy data on men with more than one follow-up biopsy. Am J Surg Pathol. 2001;25(8):1079-1085.

26. Stewart CS, Leibovich BC, Weaver AL, Lieber MM. Prostate cancer diagnosis using a saturation needle biopsy technique after previous negative sextant biopsies. J Urol. 2001;166(1):86-91.

27. Fowler JE, Bigler SA, Miles D, Yalkut DA. Predictors of first repeat biopsy cancer detection with suspected local stage prostate cancer. J Urol. 2000;163(3):813-818.

28. Iczkowski KA, Chen HM, Yang XJ, Beach RA. Prostate cancer diagnosed after initial biopsy with atypical small acinar proliferation suspicious for malignancy is similar to cancer found on initial biopsy. Urology. 2002;60(5):851-854.

29. O'dowd GJ, Miller MC, Orozco R, Veltri RW. Analysis of repeated biopsy results within 1 year after a noncancer diagnosis. Urology. 2000;55(4): 553-559.

30. Park S, Shinohara K, Grossfeld GD, Carroll PR. Prostate cancer detection in men with prior high grade prostatic intraepithelial neoplasia or atypical prostate biopsy. J Urol. 2001;165 (5): 1409-1414.

31. Borboroglu PG, Sur RL, Roberts JL, Amling CL. Repeat biopsy strategy in patients with atypical small acinar proliferation or high grade prostatic intraepithelial neoplasia on initial prostate needle biopsy. J Urol. 2001;166(3):866-870.

32. Iczkowski KA, Bassler TJ, Schwob VS, Bassler IC, Kunnel BS, Orozco RE, et al: Diagnosis of "suspicious for malignancy" in prostate biopsies predictive value for cancer. Urology. 1998;51 (5):749-757.

33. Allen EA, Kahane H, Epstein JI. Repeat biopsy strategies for men with atypical diagnoses on initial prostate needle biopsy. Urology. 1998;52(5):803-807.

Correspondencia autor: Dr. C. Pascual Mateo

Servicio de Urología. Hospital Infanta Cristina

Toledo, s/n - Parla (Madrid)

Tel.: 911913000

E-mail autor: carlospascualmateo@yahoo.es

Información artículo: Original - Cáncer de próstata

Trabajo recibido: octubre 2007

Trabajo aceptado: marzo 2008 